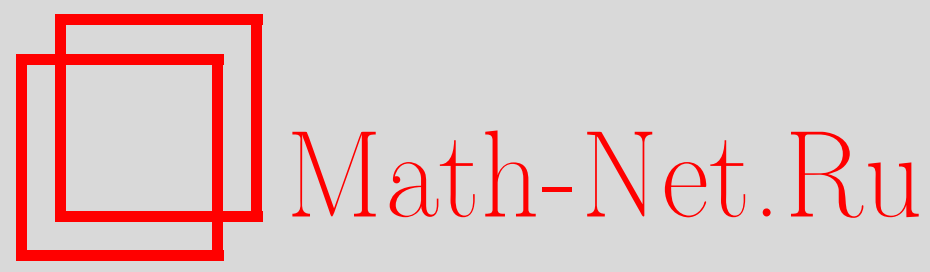

О. Н. Агеев, О функции кратности спектра динамических систем, Матем. заметки, 1999, том 65, выпуск 4, 619-622

DOI: https://doi.org/10.4213/mzm1089

Использование Общероссийского математического портала Math-Net.Ru подразумевает, что вы прочитали и согласны с пользовательским соглашением http://www. mathnet.ru/rus/agreement

Параметры загрузки:

IP: 54.196.121.252

26 апреля 2023 г., 18:09:35

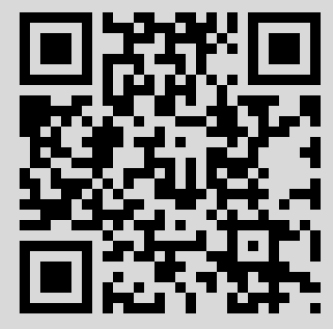


ТОм 65 выпУСК 4 АпРель 1999

\author{
КРАТКИЕ СООБЩЕНИЯ
}

\title{
О ФУНКЦИИ КРАТНОСТИ СПЕКТРА ДИНАМИЧЕСКИХ СИСТЕМ
}

\section{O.Н. Агеев}

В статье рассматриваются сохраняющие непрерывную меру $\mu$ преобразования $T$ пространства Лебега $(X, \mu), \mu(X)=1$, называемые динамическими системами. Будем обозначать индуцированные ими унитарные операторы в $L_{2}(\mu), T f(x)=f(T x)$, теми же символами, группу всех преобразований через $\operatorname{Aut}(\mu)$, множество существенных значений функции кратности спектра унитарного оператора $U$ через $M(U)$.

В литературе [1], [2] не раз отмечалась сложность одной из классических нерешенных проблем спектральной теории преобразований с инвариантной мерой: указать точные условия на спектр унитарного оператора такие, что он мог быть реализован эргодической динамической системой. Не решены даже частные случаи этой задачи: существование преобразования с простым лебеговским спектром (проблема Банаха) и с конечнократным абсолютно непрерывным спектром в ортогональном дополнении к пространству констант $\{f: f \equiv c\}^{\perp}$ (см. [2]).

Очевидно, $M(U) \subseteq \mathbb{N} \cup\{\infty\}$. Отметим, что $M(T)$ может быть любым подмножеством из $\mathbb{N} \cup\{\infty\}$, если не требовать эргодичности преобразования $T$. Для эргодических преобразований $T$ имеем $1 \in M(T)$, где 1 отвечает циклическому подпространству $\left\{f \in L_{2}(\mu): T f=f\right\}$. По реализации подмножеств из $\mathbb{N} \cup\{\infty\}$ в виде $M(T)$ для эргодических преобразований $T$ (отличных от $\{1\}$ и $\{1, \infty\})$ отметим работы [3]-[6]. В [3] построены первые примеры эргодических преобразований с $M(T)=\{1,2\}$. В [4] для любого натурального $n$ построены преобразования $T$ с $M(T)=\{1, n\}$. В [5], [6] последовательно расширялся класс подмножеств из $\mathbb{N}$, реализуемых как $M(T)$. Заметим, что эти конструкции существенно использовали наличие в централизаторе преобразования $T$ косого произведения с групповым автоморфизмом, дающее следующее ограничение на $M(T): m_{1}, m_{2} \in M(T) \Longrightarrow \operatorname{HOK}\left(m_{1}, m_{2}\right) \in M(T)$. Предложенная ниже конструкция динамических систем, в частности, позволяет снять это ограничение и показывает, что типичное преобразование $T$ из $\operatorname{Aut}(\mu)$ для любого $M \subseteq \mathbb{N} \cup\{\infty\}$, где $1 \in M$, допускает эргодические групповые расширения $\widetilde{T}$ с $M(\widetilde{T})=M$. Методика доказательства не использует групповые автоморфизмы и по духу близка методу геометрических моделей, примененных к изучению спектральных свойств классических преобразований фон Неймана (см. [7], [8]).

Заметим, что принято рассматривать спектр преобразований в $\{f: f \equiv c\}^{\perp}$. В значительной мере интерес здесь обусловлен нерешенной задачей Рохлина о сушествовании эргодического преобразования с $M(T)=\{n\}$, где $n \notin\{1, \infty\}$ (см. [4], [9]).

Работа выполнена при частичной финансовой поддержке программы "Ведущие научные школы”, грант № 96-15-96135, и совместного проекта Российского фонда фундаментальных исследований и фонда INTAS, грант № 00-418i96. 
1. Определение класса $A$. Рассмотрим кусочно-линейные, сохраняющие ориентацию преобразования на $[0,1)$ с мерой Лебега $\mu$. Преобразование $T$ из класса $A$ удобно определить, достраивая рекуррентно: на $k$-м шаге имеем представление $[0,1)$ в виде дизъюнктного объединения полуинтервалов $I_{1}, I_{2}, \ldots, I_{q_{k}}, I_{k}^{\prime}$, где $I_{k}^{\prime}$ крайний справа, полуинтервалы $I_{i}\left(1 \leqslant i \leqslant q_{k}\right)$ имеют одну длину и $T$ отображает линейно $I_{i}$ на $I_{i+1}\left(1 \leqslant i<q_{k}\right)$. Преобразование $T$ отображает $I_{q_{k}} \cup I_{k}^{\prime}$ на $I_{k}^{\prime} \cup I_{1}$, но неопределено на этом шаге. Опишем переход от $k$ к $k+1$. Фиксируем натуральное число $p_{k}$ и последовательность неотрицательных целых чисел $\left\{a(k, p): 1 \leqslant p \leqslant p_{k}\right\}$. Разобьем каждый $I_{i}$ на $p_{k}$ полуинтервалов $I_{i, p}\left(1 \leqslant p \leqslant p_{k}\right)$ равной длины, занумерованных слева направо. Ясно, что $T$ отображает линейно $I_{i, p}$ на $I_{i, p+1}\left(1 \leqslant i<q_{k}\right)$. Разобьем $I_{k}^{\prime}$ на полуинтервалы $I_{k, p, a}^{\prime}(p \in\{p: a(k, p)>0\}, 1 \leqslant a \leqslant a(k, p))$ одной длины, совпадающей с длиной $I_{i, p}$, и крайний справа полуинтервал $I_{k+1}^{\prime}$. На $(k+1)$-м шаге имеем представление $[0,1)$ в виде дизъюнктного объединения полуинтервалов

$$
\left\{I_{k+1}^{\prime}\right\} \cup\left\{I_{i, p}: 1 \leqslant i \leqslant q_{k}, 1 \leqslant p \leqslant p_{k}\right\} \cup\left\{I_{k, p, a}^{\prime}: p \in\{p: a(k, p)>0\}, 1 \leqslant a \leqslant a(k, p)\right\}
$$

и

$$
q_{k+1}=q_{k} p_{k}+\sum_{p} a(k, p)
$$

Доопределим преобразование $T$ (с сохранением ориентации), переставляя полуинтервалы по следующей схеме:

$$
\begin{gathered}
I_{q_{k}, p} \rightarrow I_{1, p+1} \quad \text { при } p<p_{k} \text { и } a(k, p)=0, \\
I_{q_{k}, p} \rightarrow I_{k, p, 1}^{\prime} \rightarrow I_{k, p, 2}^{\prime} \rightarrow \cdots \rightarrow I_{k, p, a(k, p)}^{\prime} \rightarrow I_{1, p+1} \quad \text { при } p<p_{k} \quad \text { и } a(k, p)>0, \\
I_{q_{k}, p_{k}} \rightarrow I_{k, p_{k}, 1}^{\prime} \rightarrow \cdots \rightarrow I_{k, p_{k}, a\left(k, p_{k}\right)}^{\prime} \quad \text { при } a\left(k, p_{k}\right)>0 .
\end{gathered}
$$

Ясно, что $I_{q_{k+1}}$ есть $I_{k, p_{k}, a\left(k, p_{k}\right)}^{\prime}$ при $a\left(k, p_{k}\right)>0$ и $I_{q_{k}, p_{k}}$ в противном случае. Преобразование $T$ отображает $I_{k+1}^{\prime} \cup I_{q_{k+1}}$ на $I_{1} \cup I_{k+1}^{\prime}$, но неопределено на $(k+1)$-м шаге.

Рассмотренная выше конструкция есть требуемая модификация представления любого преобразования, имеющего ранг 1. Потребуем дополнительно, чтобы для некоторой последовательности номеров $k_{n} \rightarrow \infty$ выполнялось:

$$
\begin{aligned}
& p_{k_{n}} \rightarrow \infty, \quad a\left(k_{n}, p\right)=0, \quad 1 \leqslant p \leqslant p_{k_{n}}, \\
& \frac{\mu\left(T I_{q_{k_{n}}, p_{k_{n}}} \cap I_{1,1}\right)}{\mu\left(I_{1,1}\right)} \rightarrow 1 \quad \text { при } n \rightarrow \infty .
\end{aligned}
$$

Отметим, что построенный класс $A$ является подмножеством жестких (т.е. $T \stackrel{r_{n}}{\rightarrow} E$ при $r_{n} \rightarrow \infty$, где $E$ - тождественный оператор) преобразований ранга 1.

2. Конструкция косых произведений. Фиксируем $M=\left\{1, n_{1}, n_{2}, \ldots\right\} \subseteq \mathbb{N}$ и преобразование $T$ из класса $A$. Введем обозначения

$$
\begin{gathered}
\tilde{N}=\sum_{j} n_{j}, \quad \tilde{N} \leqslant \infty, \quad l=\#\left\{n_{1}, n_{2}, \ldots\right\}, \quad l \leqslant \infty \\
\widetilde{N}-l=\sum_{j}\left(n_{j}-1\right), \quad \Omega=\mathbb{T}^{l} \times C(T)^{\tilde{N}-l}
\end{gathered}
$$

где $\mathbb{T}$ - окружность, естественным образом отождествленная с $[0,1), C(T)=\{S \in \operatorname{Aut}(\mu):$ $T S=S T\}$. 
Положим

$$
\omega_{\gamma}(x)=\left\{\begin{array}{ll}
-1, & 0 \leqslant x<\gamma, \\
1, & \gamma \leqslant x<1,
\end{array} \quad \gamma, x \in \mathbb{T} \equiv[0,1) .\right.
$$

Далее, для удобства, будем использовать нумерацию координат в $\Omega$, учитьвающую структуру множества $M$. Сопоставим каждому $\widetilde{\beta} \in \Omega$, где $\widetilde{\beta}=\left(\beta_{1}, \beta_{2}, \ldots, S_{1,1}, S_{1,2}, \ldots, S_{1, n_{1}-1}, S_{2,1}\right.$, $\left.S_{2,2}, \ldots, S_{2, n_{2}-1}, \ldots\right)\left(S_{i, j} \in C(T), \beta_{i} \in \mathbb{T}\right)$ вектор-функцию $\omega_{\widetilde{\beta}}(x)=\left(\omega_{\beta_{1}}(x), \omega_{\beta_{1}}\left(S_{1,1} x\right), \ldots\right.$, $\left.\omega_{\beta_{1}}\left(S_{1, n_{1}-1} x\right), \omega_{\beta_{2}}(x), \omega_{\beta_{2}}\left(S_{2,1} x\right), \ldots\right)$ и преобразование $T_{\omega_{\widetilde{\beta}}}$, действующее в пространстве $\mathbb{T} \times$ $\mathbb{Z}_{2}^{\tilde{N}} \equiv[0,1) \times\{-1,1\}^{N}$ с мерой Хаара $m=\mu \times l \times l \times \cdots(l\{i\}=0.5, i \in\{-1,1\})$ по формуле $T_{\omega_{\widetilde{\beta}}}(x, y)=\left(T x, \omega_{\widetilde{\beta}}(x) * y\right)$, где $*-$ покоординатное умножение.

Заметим, что $C(T)$, как замкнутое подмножество в $\operatorname{Aut}(\mu)$ относительно слабой топологии, есть польское (полное метризуемое сепарабельное) пространство. Поэтому $\Omega$ есть польское пространство относительной топологии декартова произведения.

Говорим, что для типичного элемента из $A$ имеет место некоторое свойство, если множество элементов из $A$, удовлетворяющих этому свойству, содержит плотное подмножество типа $G_{\delta}$ в $A$ (а следовательно, второй категории для рассматриваемых в статье пространств).

Теорема 1. Для любого әлемента $T$ из класса $A$ для типичного $\widetilde{\beta} \in \Omega$ преобразования $T_{\omega_{\widetilde{\beta}}}$ әргодичнь, $M\left(T_{\omega_{\widetilde{\beta}}}\right)=M$ имножества собственнъх функиий преобразований $T$ $u T_{\omega_{\tilde{\beta}}}$ совпадают.

3. Модельные реализации. Рассматриваемый ниже вариант аппроксимаций преобразований пространства Лебега $(X, \mu)$ периодическими преобразованиями (см. [9]) естественно диктуется задачей, хотя, формально, ранее в литературе не встречался.

Говорим, что преобразование $T$ допускает попарную аппроксимачию плоскими башнями, если существует последовательность $\left\{\xi_{k}\right\}$ разбиений $X$ такая, что

$$
\begin{gathered}
\xi_{k}=\left\{B^{\prime}(k), B_{0}(k), T B_{0}(k), \ldots, T^{q_{k}-1} B_{0}(k)\right\}, \quad \xi_{k} \rightarrow \varepsilon, \\
\xi_{2 k} \leqslant \xi_{2 k+1}, \quad k \geqslant 0, \quad \frac{q_{2 k+1}}{q_{2 k}}=p_{k} \in \mathbb{N}, \\
\mu\left(B_{0}(2 k) \mid T^{n q_{2 k}} B_{0}(2 k+1)\right)=1, \quad 0 \leqslant n<p_{k}, \\
\mu\left(B_{0}(2 k) \mid T^{p_{k} q_{2 k}} B_{0}(2 k+1)\right) \rightarrow 1 \quad \text { при } k \rightarrow \infty, \\
p_{k} \rightarrow \infty \quad \text { при } k \rightarrow \infty, \quad \mu\left(B_{0}(2 k)\right)=p_{k} \mu\left(B_{0}(2 k+1)\right) .
\end{gathered}
$$

Заметим, отсюда следует, что $\mu\left(B_{0}(2 k+1) \mid T^{p_{k} q_{2 k}} B_{0}(2 k+1)\right) \rightarrow 1$ при $k \rightarrow \infty$.

Лемма 1. Типичный әлемент из $\operatorname{Aut}(\mu)$ относительно слабой топологии допускает попарную аппроксимацию плоскими башнями.

ЛЕмма 2. Преобразование, допускающее попарную аппроксимачию плоскими башнями, метрически изоморфно преобразованию из класса $A$.

Ясно, что из лемм 1 и 2 с учетом теоремы 1 следует основной результат.

Tеорема 2. Для типичного әлемента $T$ из $\operatorname{Aut}(\mu)$ относительно слабой топологии и типичного $\widetilde{\beta} \in \Omega=\mathbb{T}^{l} \times C\left(\varphi_{T} T \varphi_{T}^{-1}\right)^{\tilde{N}-l}$ мнохества собственных функций преобразований $T$ и $\widetilde{T}$ совпадают и $M(\widetilde{T})=M$, əде $\widetilde{T}(x, y)=\left(T x, \omega_{\widetilde{\beta}}\left(\varphi_{T}(x)\right) * y\right)$ действует в $X \times \mathbb{Z} \tilde{N}^{\widetilde{N}}$, $\varphi_{T}(x): X \rightarrow \mathbb{T}-$ изоморфизм такой, что $\varphi_{T} T \varphi_{T}^{-1} \in A$. 
ЗАМЕчАнИЕ 1 . Нетрудно достроить любое преобразование $\widetilde{T}$, используя стандартную процедуру декартова произведения, до преобразования $\widetilde{\widetilde{T}}$, имеющего тот же набор собственных функций, что и $\widetilde{T}$, так что $M(\widetilde{\widetilde{T}})=M(\widetilde{T}) \cup\{\infty\}$.

ЗАмЕчАниЕ 2. Для типичных вектор-функций $\omega(x)$ относительно слабой топологии и типичного преобразования $T$ имеем $M(\widetilde{T})=\{1\}$ (а значит, $\widetilde{T}$ эргодично), где $\widetilde{T}(x, y)=(T x, \omega(x) * y)$.

Преобразование, допускающее попарную аппроксимацию плоскими башнями, вообще говоря, не обязано иметь непрерьвный спектр. Однако, преобразования с непрерывным спектром типичны в $\operatorname{Aut}(\mu)$. В итоге имеем

СледствиЕ. Типичнъй элемент $T$ в әруппе $\operatorname{Aut}(\mu)$ для любого множества $M \subseteq \mathbb{N} \cup$ $\{\infty\}(1 \in M)$ имеет әрупповое расширение $\widetilde{T}$ с непрерывным спектром в пространстве $\{f: f \equiv c\}^{\perp}$ maкое, ито $M(\widetilde{T})=M$.

\section{СПИСОК ЦИТИРОВАННОЙ ЛИТЕРАТУРЫ}

1. Рохлин В. А. // УМН. 1967. Т. 22. № 5. С. 3-56. 2. Вершик А. М., Корнфельд И. П., Синай Я. Г. // Итоги науки и техн. Соврем. пробл. матем. Фундамент. направления. Т. 2. М.: ВИНИТИ, 1985. С. 5-111. з. Оселедец В. И. // Докл. АН СССР. 1966. Т. 168. № 5. С. 1009-1011. 4. Robinson E. A. // Invent. Math. 1983. V. 72. P. 299-314. 5. Robinson E. A. // Israel J. Math. 1986. V. 56. №1. P. 75-88. 6. Goodson G. R., Kwiatkowski I., Lemanczyk M., Liardet P. // Studia Math. 1992. V. 102. № 2. P. 157-174. 7. Агеев О. Н. // УМН. 1994. Т. 49. №2. С. 143-144. 8. Агеев О. Н. // Матем. сб. 1997. Т. 188. №8. С. 13-44. 9. Каток А. Б., Синай Я. Г., Стёпин А. М. // Итоги науки и техн. Матем. анализ. Т. 13. М.: ВИНИТИ, 1975. C. $129-262$.

Московский государственный технический университет им. Н. Э. Баумана

Поступило

E-mail : ageev@mx.bmstu.ru

01.10 .98 